\title{
FREQUENCY OF DEPRESSION AND ANXIETY SYMPTOMS IN MEXICAN PATIENTS WITH RHEUMATIC DISEASES DETERMINED BY SELF-ADMINISTERED QUESTIONNAIRES ADAPTED TO THE SPANISH LANGUAGE
}

\author{
Luis F. Pérez-García ${ }^{1}$, Luis H. Silveira ${ }^{1}$, Mariana Moreno-Ramírez ${ }^{1}$, Javier Loaiza-Félix ${ }^{1}$, Vijaya Rivera ${ }^{1}$ \\ AND LUIS M. AMEZCUA-GUERRA $2,3,4$ \\ Departments of ${ }^{1}$ Rheumatology and ${ }^{2}$ Immunology, Instituto Nacional de Cardiología Ignacio Chávez, Mexico City; \\ ${ }^{3}$ Department of Health Care, Universidad Autónoma Metropolitana - Xochimilco; ${ }^{4}$ Cardiovascular Research Laboratory, \\ Unidad de Investigación Traslacional, Universidad Nacional Autónoma de México/Instituto Nacional de Cardiología \\ Ignacio Chávez, Mexico City, Mexico
}

\begin{abstract}
Background: The frequency of depression and anxiety symptoms in Spanish-speaking patients suffering from rheumatic conditions is unknown when using self-administered detection tools. Methods: A single-center, cross-sectional survey including 413 patients (341 women) with well-defined rheumatic diseases was conducted. The patient health questionnaire-9 (PHQ-9) and generalized anxiety disorder (GAD)-7 questionnaires were used to detect depression and anxiety symptoms, respectively. Results: A total of 193 patients (46.7\%) reported depression symptoms, and increased PHQ-9 scores were more frequently observed in women than in men ( $23 \%$ vs. $13 \% ; p=0.038$ ), particularly in association with osteoarthritis, fibromyalgia, Sjögren's syndrome, and osteoporosis. From 88 patients (21.3\%) with PHQ-9 scores $\geq 10$ points (moderate-to-severe depression symptoms), 27 (30.6\%) were previously diagnosed to have depression and only four were under antidepressant treatment. Anxiety symptoms were observed in 168 patients (40.6\%) and classified as moderate-to-severe by elevated GAD-7 scores in 68 subjects (16.4\%). Of them, 12 (17.6\%) were previously diagnosed with GAD, but only 4 (5.8\%) were under therapy. Conclusions: An unexpected and unusually high frequency of undiagnosed depression and anxiety symptoms was found in rheumatic patients. Self-administered screening tools adapted to the Spanish language are useful and may help clinicians to suspect these conditions. (REV INVEST CLIN. 2019;71:91-7)
\end{abstract}

Key words: Depression. Anxiety. Mental health questionnaires. Rheumatic diseases.

Corresponding author:

*Luis M. Amezcua-Guerra

Immunology and Cardiovascular Research Laboratory Instituto Nacional de Cardiología Ignacio Chávez

Juan Badiano, 1

Col. Sección XVI, Del. Tlalpan

C.P. 14080, Mexico City, Mexico

Received for publication: 06-08-2018

E-mail: Imamezcuag@gmail.com

Approved for publication: 03-11-2018

DOI: $10.24875 / R I C .18002698$ 


\section{INTRODUCTION}

Depression and generalized anxiety disorder (GAD) are comorbid conditions frequently found in patients with rheumatic diseases. A recent study found a prevalence of $21.8 \%$ for depression and $37 \%$ for anxiety, in 514 Greek patients with rheumatic conditions ${ }^{1}$. Notably, depression and anxiety had not been previously diagnosed in most of these patients; therefore, they were not under medical treatment. Recognition of a mental health disorder is essential to receive adequate therapy, and it is estimated that only $50 \%$ of patients with depression are diagnosed $^{2}$. In this regard, several factors may be responsible for the high frequency of undiagnosed depression and anxiety in patients with rheumatic diseases including a persistent social stigma surrounding mental illness (both among physicians and patients), poor communication between patients and physicians, and the limited time available in outpatient clinics to explore properly the existence of mental health disorders ${ }^{3}$. This has important implications in rheumatic patients since depression and anxiety have been associated with a poorer quality of life, higher disease activity, more pain, greater fatigue, worse outcomes, longer time to achieve remission, a decrease in response to treatment and in the likelihood of remission, increased costs, and increased mortality ${ }^{1,4,5}$.

Recently, the European League Against Rheumatism proposed several elements aimed to evaluate comorbidities in patients with rheumatic diseases, specifically considering depression as a comorbidity that should be examined using a simple question, without referring to any specific screening tool ${ }^{6}$. To improve the detection of mental health disorders, there are some self-administered tools that are quick and easy to complete, saving valuable consultation time. These instruments may also minimize social stigma and have been extensively validated to detect patients with clinical depression and anxiety. However, most of these tools have been developed in English-speaking countries, which create problems when used in regions with other languages and cultures. Spanish is the most widely spoken language in the world after Mandarin Chinese, and the number of speakers is rising; therefore, there is a need to apply these instruments in patients who speak a language other than English to evaluate whether transcultural adaptation certainly ensures that these instruments mean the same as in their original version ${ }^{7,8}$.

In this study, we sought to investigate the frequency of the symptoms of depression and anxiety in Mexican patients with rheumatic diseases, using self-administered questionnaires already adapted to the Spanish language, as well as to evaluate their usefulness in a real-world setting.

\section{METHODS}

Adult patients who attended our outpatient rheumatology clinic (a public tertiary care practice in a university setting) from March to June 2016 were invited to participate in this cross-sectional survey. Patients were asked to participate if they had an established diagnosis of a rheumatic disease. We excluded individuals who, in the investigator's judgment, were unable to understand or answer the questions included in the questionnaires due to their educational level or lack of knowledge of the Spanish language. Each diagnosis was verified by attending physicians and only patients who met the current classification criteria for their respective rheumatic disease were included. A medical history was obtained, including demographic and clinical data with a special focus on the previous establishment of a psychiatric diagnosis. Socioeconomic status was obtained from the medical record; in our hospital, this represents a composite of the degree of education, family income, and occupation, and is routinely evaluated by a professional social worker. The family status was also obtained from the medical record and classified in a dichotomous way as married/living with a partner or not. In the understanding of the heterogeneity of rheumatic diseases included in the study and considering the wide diversity of therapeutic schemes and drugs used for each one, information related to treatment, functional ability, and disease activity was not collected. All participants were Mexican Mestizo (mixed European and Amerindian ancestry).

The study protocol and data collection were approved by the local ethics committee. All patients consented to provide their data for research purposes. All procedures were performed in accordance with the Declaration of Helsinki and local regulations. 
Table 1. Main demographic and clinical characteristics of the study population.

\begin{tabular}{|c|c|}
\hline & $\begin{array}{l}\text { Study participants } \\
\qquad(\mathrm{n}=413)\end{array}$ \\
\hline Age in years, mean $\pm S D$ & $50.8 \pm 16.2$ \\
\hline Female gender, n (\%) & $341(82.5)$ \\
\hline Socioeconomic status ${ }^{a}$ & 396 \\
\hline Low-income, n (\%) & $352 / 396(88.9)$ \\
\hline Middle-income, n (\%) & $38 / 396(9.6)$ \\
\hline High-income, n (\%) & $6 / 396(1.5)$ \\
\hline Married or living with a partner, n $(\%)^{a}$ & $279 / 371(75.2)$ \\
\hline Previous depression diagnosis, n (\%) & $27(6.5)$ \\
\hline PHQ-9 scale, median score with IQR & $4(2-8)$ \\
\hline No depression, n (\%) & $220(53.2)$ \\
\hline Mild depression symptoms, n (\%) & $105(25.4)$ \\
\hline Moderate depression symptoms, n (\%) & $56(13.5)$ \\
\hline Severe depression symptoms, n (\%) & $32(7.7)$ \\
\hline Previous anxiety diagnosis, n (\%) & $12(2.9)$ \\
\hline GAD-7 scale, median score with IQR & $3(1-7)$ \\
\hline No anxiety, n (\%) & $245(59.3)$ \\
\hline Mild anxiety symptoms, n (\%) & $100(24.2)$ \\
\hline Moderate anxiety symptoms, n (\%) & $43(10.4)$ \\
\hline Severe anxiety symptoms, n (\%) & $25(6)$ \\
\hline
\end{tabular}

SD: standard deviation, IQR: interquartile range.

aDue to missing data in medical records, participants may not add up to 413

Depression was assessed with the Spanish language version of the patient health questionnaire-9 (PHQ-9) test, which contains 9 items scored from 0 to $27^{9}$. It also includes a question to assess whether depression symptoms are affecting functioning, a key criterion for establishing a diagnosis based on the Diagnostic and Statistical Manual of Mental Disorders (DSM)-5. This instrument is considered more accurate than other screening tools, with $88 \%$ sensitivity and $88 \%$ specificity ${ }^{10}$. Anxiety was evaluated with the 7-item GAD-7 scale, a questionnaire based on 7 items, which has also been validated in the Spanish language ${ }^{11}$. It is a useful tool with strong criterion validity to identify individuals with a possible GAD. In addition, the instrument is an excellent measure of disease severity, as demonstrated by the fact that increasing scores in GAD-7 scale are strongly associated with multiple domains of functional impairment and the number of disability days ${ }^{12}$. In this study, we used the
Spanish language versions of PHQ-9 and GAD-7 originally translated in Spain, without making any crosscultural adaptation ${ }^{9,11}$. It should be noted that these versions have already been used and validated in Latin American individuals ${ }^{13,14}$. All participants selfcompleted the PHQ-9 and GAD-7 formats in the waiting room of our rheumatology clinic.

PHQ-9 scores were classified as not elevated (score $0-4$ ), mildly elevated (5-9), moderately elevated (10$14)$, or severely elevated ( $\geq 15)$, and these should be interpreted as representing no depression, mild, moderate, and severe levels of depression, respectively. The same cutoff points were used to measure the levels of GAD for GAD-7 test.

\section{Statistical analysis}

Continuous variables were expressed as means \pm standard deviations or medians with interquartile range, while frequencies were used to describe discrete variables. Differences were assessed using the Student's t-test or the Pearson Chi-square test with Yates' correction, as corresponded. $p<0.05$ value was set for statistical significance. Analyses were performed using GraphPad Prism version 7.00 for Windows, GraphPad Software, La Jolla, California, USA.

\section{RESULTS}

A total of 413 study subjects ( 341 women; $82.5 \%$ ) were recruited. Table 1 shows a summary of the main clinical data. On average, patients included in this analysis were 50 years old, married or living with a partner, and had a low-to-medium socioeconomic level. The main rheumatic conditions were rheumatoid arthritis, systemic lupus erythematosus, fibromyalgia, and osteoarthritis.

Overall, 57 (13.8\%) patients did not score any point in any of the items of PHQ-9 questionnaire (PHQ-9 score $=0$ ). In $193(46.7 \%)$ patients, PHQ-9 scores were $\geq 5$. We found PHQ-9 scores $\geq 10$ (considered to be moderately to severely elevated) more frequently in women than in men ( $23 \%$ vs. $13 \% ; p=0.038$ ). Interestingly, from a total of 88 (21.3\%) subjects showing moderate and severe elevations of PHQ-9 scores ( $\geq 10$ points), 27 (30.6\%) had been previously diagnosed as having depression and only $4(4.5 \%)$ 
Figure 1 . Frequency and severity of depression symptoms in 413 rheumatic patients according to the patient health questionnaire- 9 scale. The bars represent the number of participants suffering from each rheumatic disease.

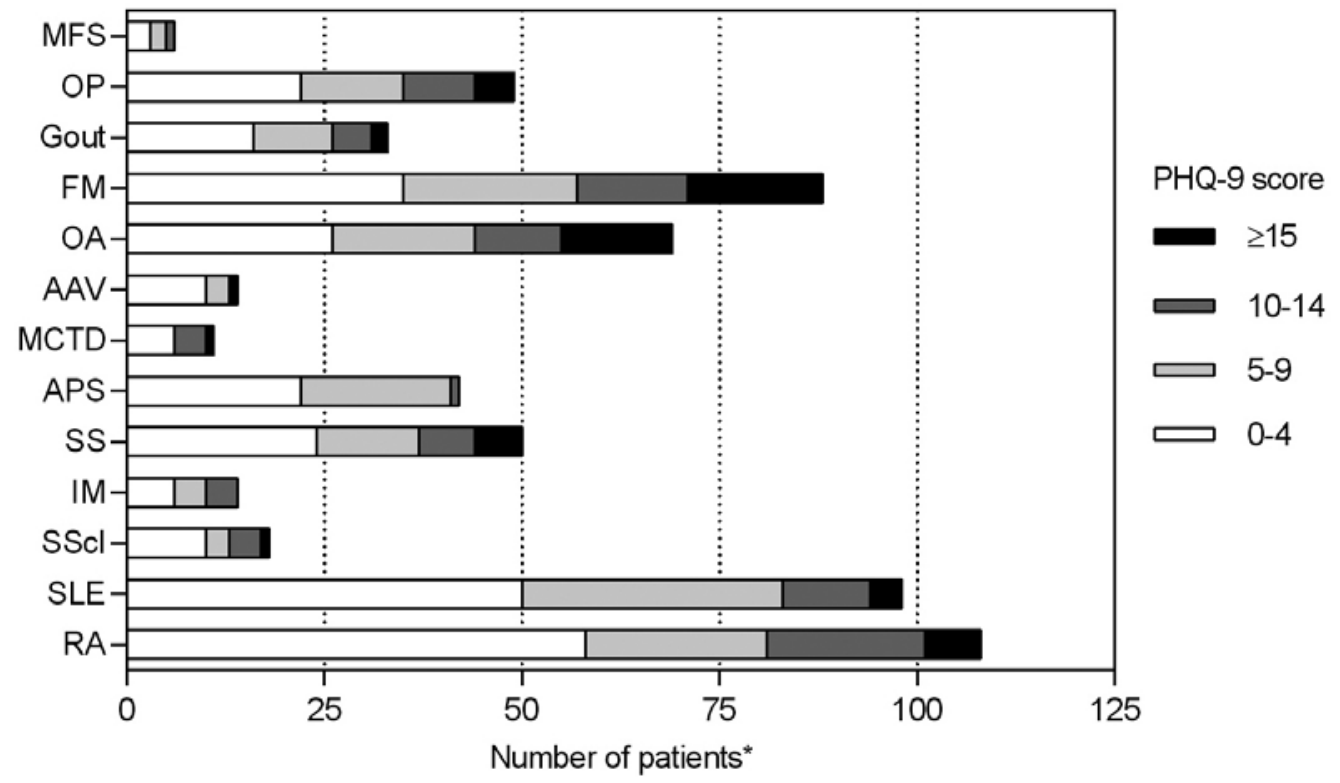

MFS: Marfan syndrome; OP: osteoporosis; FM: fibromyalgia; OA: osteoarthritis; AAV: ANCA-associated vasculitis; MCTD: mixed connective tissue disease; APS: antiphospholipid syndrome; SS: Sjögren's syndrome; IM: inflammatory myopathies; SScl: systemic sclerosis;

SLE: systemic lupus erythematosus; RA: rheumatoid arthritis.

*Patients could have more than one concurrent rheumatic disease, so the total sum is greater than the total number of subjects included in the study.

were under therapy with antidepressants. Figure 1 shows a summary of the distribution and severity of depression symptoms according to PHQ-9 scale, by rheumatic condition. As noted, the highest frequencies of the severe elevations of PHQ-9 scale were observed in patients with osteoarthritis (20.2\%), fibromyalgia (19.3\%), Sjögren's syndrome (12\%), and osteoporosis (10.2\%).

91 patients (22\%) did not score any point in any of the items of GAD-7 questionnaire (GAD-7 score $=0$ ). Generalized anxiety symptoms (GAD-7 score $\geq 5$ ) were found in 168 (40.6\%) subjects, 68 of them showing moderate-to-severe elevations of GAD-7 scores ( $\geq 10$ points). Of these patients classified as having moderate or severe symptoms of anxiety, 12 (17.6\%) were previously diagnosed as having GAD and only $4(5.8 \%)$ were under medical treatment. Figure 2 shows a summary of the distribution and severity of anxiety symptoms according to GAD-7 scale, by rheumatic condition. As noted, the highest frequency of patients having severe elevations of GAD-7 score was found in patients with fibromyalgia (12.5\%) and osteoarthritis (11.5\%).
An overlap of symptoms compatible with depression and generalized anxiety was observed in 90 patients with rheumatic conditions, which represents $21.7 \%$ of the study participants.

\section{DISCUSSION}

The findings of this cross-sectional survey suggest that self-administered screening tools are very useful and may help clinicians to suspect undiagnosed depression and/or GAD in patients suffering from rheumatic diseases. In addition, there was an unusually high rate of rheumatic patients with depression $(46.7 \%)$ and anxiety (40.6\%) symptoms; surprisingly, most patients had not been previously diagnosed with a mental illness despite receiving medical care regularly.

Depression is a major cause of ill health and disability worldwide, and, at worst, may lead to suicide. According to the latest estimates from the World Health Organization (WHO), more than 300 million people are now living with depression, which represents an 
Figure 2. Frequency and severity of generalized anxiety symptoms in 413 rheumatic patients according to the generalized anxiety disorder-7 scale. The bars represent the number of participants suffering from each rheumatic disease.

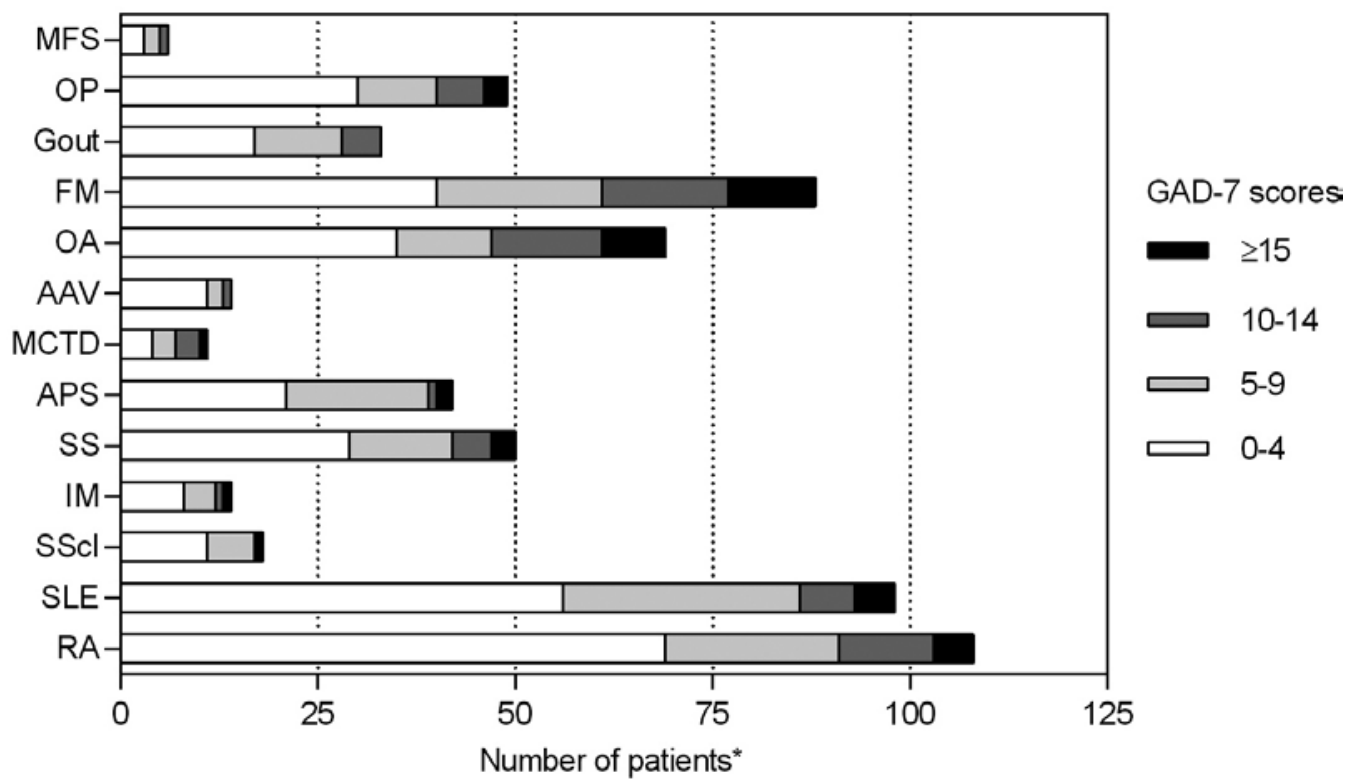

MFS: Marfan syndrome; OP: osteoporosis; FM: fibromyalgia; OA: osteoarthritis; AAV: ANCA-associated vasculitis; MCTD: mixed connective tissue disease; APS: antiphospholipid syndrome; SS: Sjögren's syndrome; IM: inflammatory myopathies; SScl: systemic sclerosis; SLE: systemic lupus erythematosus; RA: rheumatoid arthritis.

*Patients could have more than one concurrent rheumatic disease, so the total sum is greater than the total number of subjects included in the study.

increase of over $18 \%$ between 2005 and $2015^{15}$. Moreover, an adequate recognition and diagnosis of depression and anxiety represent a major clinical challenge for physicians taking care of rheumatic patients, and previous studies have suggested that Latin American populations tend to have worse clinical outcomes regarding mental health disorders ${ }^{16}$. Results of the present survey are in line with studies already describing a high prevalence of depression and anxiety in rheumatic patients from other ethnicities; moreover, our findings were more than twice those found by Anyfanti et al. for depression (21.8\%) and similar to those found by them for anxiety (37\%) in Greek patients with various rheumatic diseases ${ }^{1}$. Previously, a study performed in 35 Mexican patients with rheumatoid arthritis found a $37 \%$ frequency of depression-related disorders, mainly major depression and dysthymia, using the beck depression inventory ${ }^{17}$. Recently, Matcham et al. conducted a systematic review and meta-analysis of 72 studies evaluating the prevalence of depression in rheumatoid arthritis ${ }^{12}$. They found a variation from $0.04 \%$ to $66.3 \%$, and the meta-analytical pooled prevalence according to PHQ-9 scale was $38.8 \%$, which is close to $46.2 \%$ prevalence found in our patients with rheumatoid arthritis, although they used a threshold of 10 points, indicating moderate-severe depression symptoms. In our patients with rheumatoid arthritis, the prevalence of moderate-to-severe symptoms of depression (PHQ$9 \geq 10$ points) was $25 \%$.

Of interest, the results of a study including 43,845 Mexican female teachers who responded to PHQ-9 instrument have just been released ${ }^{18}$. This study revealed that $16 \%$ of participants had PHQ- 9 score suggestive of severe depression; of them, $<5 \%$ had a formal diagnosis of depression and only $17 \%$ received antidepressants. Comparatively, of 341 female patients included in our study, 23\% had PHQ-9 score compatible with severe depression (6.5\% with a previous diagnosis of depression), and only $4.5 \%$ of them were in antidepressant treatment. Despite this, we should consider the possibility of an overestimation of depression symptoms in the subjects of our study since several of the questions included in the PHQ-9 questionnaire assess sleep and appetite disturbances, fatigue, psychomotor disorders, and concentration 
problems (see below), all of which are important issues in rheumatic patients.

Recently, the WHO launched a yearlong campaign "Depression: let's talk" on the understanding that overcoming the stigma frequently associated with depression will lead to more people getting help ${ }^{15}$. Following the WHO's campaign goals, our commitment as rheumatologists, often in the care of patients with undiagnosed depression or anxiety, should be to participate in this yearlong campaign and help to accomplish its main goal that patients with depression both seek and get help. In this regard, scales used in our study have been validated in several languages and have shown to correlate with the clinical diagnosis of depression and anxiety as well as to correlate with disease severity. On the one hand, PHQ-9 test assesses depression symptoms including anhedonia, depressed mood, insomnia or hypersomnia, fatigue, appetite and concentration disturbances, guilt, psychomotor alterations, and suicidal ideation. This instrument has been validated by classical test theory and item response theory models, and the Spanish version of PHQ-9 was shown to measure one underlying construct (i.e., depression) when used among Peruvian women ${ }^{13}$. The Spanish version of PHQ-9 has also been demonstrated to have good internal consistency in a large sample of Mexican women ${ }^{19}$. Notably, the ability of each item of PHQ-9 to capture one unique feature of depression has been shown to be independent of age, education attainment, and employment status ${ }^{13}$. On the other hand, GAD-7 questionnaire is a one-dimensional scale aimed at assessing the presence of anxiety symptoms referred in DSM-IV, and the Spanish language version has good reliability and cross-cultural validity to be used as a screening tool in Latin American individuals ${ }^{14}$. Finally, both PHQ-9 and GAD-7 instruments require a short time to be completed and may be applied by medical personnel free of charge. For instance, some studies have used the Zung Self-Rating Depression Scale for depression and the Hamilton anxiety scale for anxiety, both with more items to be answered by the patient, thus requiring more time to be completed (10-15 min, as compared to only 5-10 min for PHQ-9 and GAD-7 scales) ${ }^{1}$. Consequently, PHQ-9 and GAD-7 appear to be self-applied instruments useful for increasing the ability to detect depression and anxiety symptoms in clinically and culturally diverse settings.
The findings must be interpreted against the background of the limitations of this study. First, the cross-sectional nature of the survey does not allow us to evaluate the prognostic relevance of undiagnosed mental illnesses nor the sensitivity to change of self-administered screening tools. In the second place, there is a lack of confirmation of a diagnosis of depression or GAD by a mental health professional. Third, there is the absence of non-rheumatic patients as a control group; however, the overall prevalence of depression in Mexico has been estimated in $8 \%, 10.4 \%$ in women, and $5.4 \%$ in men ${ }^{20}$. Therefore, we can assume that our rheumatic patients have a higher frequency of depression symptoms than the general population. Finally, all participants were recruited from a single, tertiary care center, and results may not be extrapolated to a primary care setting.

Undiagnosed depression and GAD are very common in Mexican patients suffering from rheumatic diseases. Self-administered screening tools are very useful and may help clinicians to suspect depression and anxiety in rheumatology clinics for Spanishspeaking patients. The results of this study may help to change the misperception that depression and anxiety are inevitable and normal conditions in rheumatic patients. Patients and their caregivers, however, may have difficulties to deal with the increased burden of a newly diagnosed condition which will require the reconfiguration of existing service delivery models.

\section{REFERENCES}

1. Anyfanti P, Gavriilaki E, Pyrpasopoulou A, et al. Depression, anxiety, and quality of life in a large cohort of patients with rheumatic diseases: common, yet undertreated. Clin Rheumatol. 2016:35:733-9.

2. Mitchell AJ, Vaze A, Rao S. Clinical diagnosis of depression in primary care: a meta-analysis. Lancet. 2009;374:609-19.

3. Interian A, Ang A, Gara MA, Rodriguez MA, Vega WA. The longterm trajectory of depression among latinos in primary care and its relationship to depression care disparities. Gen Hosp Psychiatry. 2011;33:94-101.

4. Senra H, Rogers H, Leibach G, et al. Health-related quality of life and depression in a sample of latin american adults with rheumatoid arthritis. Int J Rheum Dis. 2017;20:1684-93.

5. Matcham F, Norton S, Scott DL, Steer S, Hotopf M. Symptoms of depression and anxiety predict treatment response and longterm physical health outcomes in rheumatoid arthritis: secondary analysis of a randomized controlled trial. Rheumatology (Oxford). 2016;55:268-78

6. Baillet A, Gossec L, Carmona L, et al. Points to consider for reporting, screening for and preventing selected comorbidities in chronic inflammatory rheumatic diseases in daily practice: a EULAR initiative. Ann Rheum Dis. 2016;75:965-73. 
7. Gómez-Valero S, García-Pérez F, Flórez-García MT, MiangolarraPage JC. Revisión sistemática de los cuestionarios autocumplimentados adaptados al español para la valoración funcional de pacientes con afecciones de rodilla. Rev Esp Cir Ortop Traumatol. 2017;61:96-103

8. Epstein J, Santo RM, Guillemin F. A review of guidelines for crosscultural adaptation of questionnaires could not bring out a consensus. J Clin Epidemiol. 2015;68:435-41.

9. Diez-Quevedo C, Rangil T, Sanchez-Planell L, Kroenke K, Spitzer RL. Validation and utility of the patient health questionnaire in diagnosing mental disorders in 1003 general hospital spanish inpatients. Psychosom Med. 2001;63:679-86.

10. Matcham F, Ali S, Irving K, Hotopf M, Chalder T. Are depression and anxiety associated with disease activity in rheumatoid arthritis? A prospective study. BMC Musculoskelet Disord. 2016; 17:155.

11. García-Campayo J, Zamorano E, Ruiz MA, et al. Cultural adaptation into spanish of the generalized anxiety disorder-7 (GAD-7) scale as a screening tool. Health Qual Life Outcomes. 2010;8:8.

12. Matcham F, Rayner L, Steer S, Hotopf M. The prevalence of depression in rheumatoid arthritis: a systematic review and meta-analysis. Rheumatology (Oxford). 2013;52:2136-48.

13. Zhong Q, Gelaye B, Fann JR, Sanchez SE, Williams MA. Crosscultural validity of the spanish version of PHQ-9 among preg- nant peruvian women: a rasch item response theory analysis. ] Affect Disord. 2014;158:148-53.

14. Zhong QY, Gelaye B, Zaslavsky AM, et al. Diagnostic validity of the generalized anxiety disorder-7 (GAD-7) among pregnant women. PLoS One. 2015;10:e0125096

15. World Health Organisation. Depression Factsheet. WHO 2017. p. 1. Available from: http://www.who.int/mediacentre/factsheets/fs369/en/. [Last accessed on 2018 Oct 24].

16. González HM, Tarraf W, Whitfield KE, Vega WA. The epidemiology of major depression and ethnicity in the united states. Psychiatr Res. 2010;44:1043-51.

17. Suárez-Mendoza AA, Cardiel MH, Caballero-Uribe CV, OrtegaSoto HA, Márquez-Marín M. Measurement of depression in Mexican patients with rheumatoid arthritis: validity of the beck depression inventory. Arthritis Care Res. 1997;10:194-9.

18. Soria-Saucedo R, Lopez-Ridaura R, Lajous M, Wirtz VJ. The prevalence and correlates of severe depression in a cohort of Mexican teachers. J Affect Disord. 2018;234:109-16.

19. Familiar I, Ortiz-Panozo E, Hall B, et al. Factor structure of the spanish version of the patient health questionnaire-9 in Mexican women. Int J Methods Psychiatr Res. 2015;24:74-82.

20. Rafful C, Medina-Mora ME, Borges G, Benjet C, Orozco R. Depression, gender, and the treatment gap in Mexico. J Affect Disord. 2012;138:165-9. 